\title{
Research Paper: Valgus Osteotomy Outcome for Treating Femoral Neck Nonunion in Young and Active Patients
}

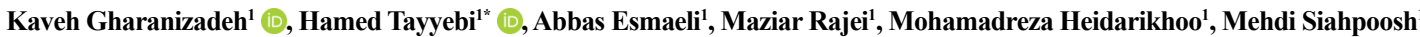

1. Bone and Joint Reconstruction Research Center, Shafa Orthopedic Hospital, Iran University of Medical Sciences, Tehran, Iran.

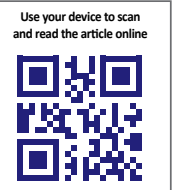

Citation Gharanizadeh K, Tayyebi H, Esmaeli A, Rajei A, Heidarikhoo MR, Siahpoosh M. Valgus Osteotomy Outcome for Treating Femoral Neck Nonunion in Young and Active Patients. Journal of Research in Orthopedic Science. 2021; 8(2):65-70. http://dx.doi.org/10.32598/JROSJ.8.2.400.1

doif : http://dx.doi.org/10.32598/JROSJ.8.2.400.1

\section{(c) (1) (8)}

Article info:

Received: 18 Mar 2021

Revised: 02 Apr 2021

Accepted: 08 Apr 2021

Available Online: 01 May 2021

Keywords:

Femoral neck fracture, Nonunion, Genu valgum, Valgus osteotomy

\begin{abstract}
A B S T R A C T
Background: Femoral neck fracture nonunion is a frequent and challenging complication, with several surgical options.

Objectives: The present study reported the outcome of valgus osteotomy for treating femoral neck nonunion in patients aged $<65$ years.

Methods: This case series research included young patients who underwent valgus osteotomy for treating femoral neck nonunion or device failure of the index surgery. The fixation of the osteotomy site was performed using either a dynamic hip screw or a fixed angle blade. The union of the neck and osteotomy site (subtrochanteric region) was evaluated by monitoring callus formation in the postoperative radiographs. Limb length discrepancy, Pauwels' angle, and the neck-shaft angle were evaluated before the surgery and at the last follow-up. Postoperative complications were extracted from the explored patients' profiles.

Results: Twenty patients with a Mean \pm SD age of $27.2 \pm 10.8$ years were examined in this study. The Mean \pm SD follow-up time of the patients was $6.1 \pm 3.9$ years. The femoral neck was united in 18 patients in a Mean \pm SD period of $5.1 \pm 2.3$ months. The Mean \pm SD limb length discrepancy was measured as $1.3 \pm 0.8 \mathrm{~cm}$ before and $0.5 \pm 0.7 \mathrm{~cm}$ after the osteotomy. The Mean \pm SD Pauwels' angle was calculated as $65.5 \pm 16.9^{\circ}$ before and $32.4 \pm 16.2^{\circ}$ after the osteotomy. The Mean \pm SD neck-shaft angle was computed to be $121.9 \pm 22.8^{\circ}$ before and $144.5 \pm 17.7^{\circ}$ after the osteotomy. Revision surgery was performed for $6(30 \%)$ patients. This measure included device removal in 4 and total hip arthroplasty in 2 patient.

Conclusion: Valgus osteotomy is a safe and efficient technique for managing femoral neck nonunion. Therefore, this approach is suggested as a good alternative for total hip arthroplasty, particularly in young and active patients.
\end{abstract}

\footnotetext{
* Corresponding Author:

Hamed Tayyebi, $M D$.

Address: Bone and Joint Reconstruction Research Center, Shafa Orthopedic Hospital, Iran University of Medical Sciences, Tehran, Iran

Phone: +98 (21) 33542041

E-mail: hamedtayebi65@gmail.com
} 


\section{Introduction}

$\mathbf{F}$

emoral Neck Fracture (FNF) is a prevalent condition accounting for approximately half of all hip fractures [1-3]. Femoral head nonunion following the fracture of the femoral neck is a major and challenging complication. Despite the improvement in surgical techniques, the rate of femoral head nonunion remains as high as $10 \%-20 \%[4]$. This rate is affected by several predisposing conditions, such as ignoring contraindications, poor fracture reduction, and inadequate internal fixation [5]. Once occurred, the available surgical options mainly include refixation, valgus osteotomy, and prosthetic replacement.

Usually, the patients are presented with limb length discrepancy, which limits the implication of refixation. Although Total Hip Arthroplasty (THA) is tempted by numerous surgeons, preserving the natural joint, particularly in young patients is beneficial. Accordingly, the valgus osteotomy is a valuable technique in treating Femoral Neck Nonunion (FNN) [4]. However, it is also associated with some disadvantages. Little bone stock in the femoral head allows moving hardware in unstable internal fixations; thus, offering too little chance for the nonunion to heal. The radiographic signs of Avascular Necrosis (AVN) in patients aged $>30$ years is a contraindication for valgus osteotomy. This is because it potentiates the occurrence of early osteoarthritis [5].

In healthy patients aged $\leq 65$ years, the valgus osteotomy is an excellent alternative for THA, as it does not limit the patients' daily living activities. Besides, it is associated with a considerably less financial burden on the healthcare system. This technique converts shearing forces into compression; accordingly, increasing the healing potential of the bone [6]. A union rate of $80 \%$ $90 \%$ was reported following the valgus osteotomy of FNN in different studies $[5,7,8]$. If valgus osteotomy provides a safe and effective treatment for FNN, it could be suggested as the method of choice for treating this complication. The study aimed to evaluate the therapeutic results of valgus osteotomy concerning treating FNN in patients aged $\leq 65$ years.

\section{Methods}

This retrospective case series study was approved by the review board of our institute. The medical profiles of patients who underwent surgical treatment for FNN at Shafa Orthopedic Hospital, Tehran City, Iran, from 2005-2019 were reviewed. Patients who were managed with valgus osteotomy were evaluated for eligibility to include in the study. The inclusion criteria of this study were the age of $<65$ years, minimum follow-up of 2 years, and no radiologic sign of AVN. Patients with metabolic bone disorders, such as osteoporosis, osteomalacia, fluorosis, primary hyperparathyroidism, and Paget's disease were excluded from the current study. Patients who were lost to follow-up were also excluded from the study. Finally, 20 patients were identified as eligible for participation in this study.

Valgus osteotomies were performed by two senior hip surgeons using a lateral approach with the patient placed on an orthopedic table. Applying subvastus approach, extracting any existing implant, the appropriate angle for inserting the new hardware was achieved by a guide pin. Then, osteotomy was performed with an oscillating saw in the subtrochanteric area (at the level of lesser trochanter). No bone graft was used. Fixation was performed using either a $130^{\circ}$ Dynamic Hip Screw (DHS, $n=5$ ) or a $130^{\circ}$ fixed angle blade $(n=15)$. The choice of fixation device was based on the surgeon's preference.

Ambulation with toe-touch was allowed in the first 6 weeks. Ambulation with partial weight-bearing was allowed after 6 weeks. Full weight-bearing was encouraged 12 weeks after the surgery. The first follow-up visit was conducted 2 weeks after the surgery. The remaining follow-ups were performed one, 2, and, 4 months after the surgery, as well as every 6 months afterward. The union of the femoral neck was evaluated by the due surgeon; the same was defined as per observing callus in the follow-up radiographs within 6 months after the primary surgery. In some cases $(n=6)$, the valgus osteotomy was administrated earlier than 6 months due to the primary device failure.

The demographic and clinical characteristics of the explored patients, including age; gender; follow-up period; limb shortening; the time interval from the injury to primary surgery and from the index surgery to valgus osteotomy, were extracted from the study patients' medical records. Postoperative complications and radiologic outcomes were also extracted from the patients' medical records. Radiologic measures included the duration of the union at the femoral neck and osteotomy site, femoral neck-shaft angle, and Pauwels' angle. The femoral neck-shaft angle was defined as the angle between the longitudinal femoral shaft axis and the femoral head-neck axis. Pauwels' angle was defined as the angle between the fracture line of the distal fragment and the horizontal line. 
SPSS v. 16 was used for analyzing the obtained data. The collected descriptive statistics were provided by Mean \pm SD as well as frequency and percentage. A Paired Samples t-test or its nonparametric counterpart (Wilcoxon signed-rank test) was used for comparing the pretest-posttest values. Moreover, $\mathrm{P}<0.05$ was considered significant.

\section{Results}

The characteristic features of the patients are demonstrated in Table 1. The study population included 15 (75\%) males and 5 (25\%) females with a Mean \pm SD age of $27.2 \pm 10.8$ years (range: $10-46 y$ ). The index treatment was surgery in 17 patients and conservative in 3 patients. Cannulated screw was used in the majority of the patients who underwent surgery (12 of 17). The Mean \pm SD follow-up period of the studied patients was $6.1 \pm 3.9$ years (range: 2-9 y).

The Mean \pm SD time interval from injury to index surgery was equal to $2.8 \pm 1.7$ days (range: $1-7 \mathrm{~d}$ ). The Mean \pm SD time interval from the index surgery to the valgus osteotomy was measured as $21 \pm 29.1$ months (range: 1-96 m). In 6 patients, the time interval from the index surgery to valgus osteotomy was $<6$ months. In these patients, valgus osteotomy was performed owing to the device failure.

The Mean \pm SD limb length discrepancy equaled $1.3 \pm 0.8$ $\mathrm{cm}$ (range: $0-3 \mathrm{~cm}$ ) before the surgery and $0.5 \pm 0.7 \mathrm{~cm}$ (range: $0-2 \mathrm{~cm})$ after the surgery $(\mathrm{P}=0.002)$. After valgus osteotomy, the union of the femoral neck occurred in 18 patients. The Mean \pm SD time interval from valgus osteotomy to the union of the femoral neck was $5.1 \pm 2.3$ months (range: 3-12 m). The union of osteotomy sites was detected in all study patients. The Mean \pm SD time interval for the union of the osteotomy site was $4.8 \pm 2.5$ months (range: 2-12 m). The Mean \pm SD Pauwels' angle was calculated as $65.5 \pm 16.9^{\circ}$ before the osteotomy and $32.4 \pm 16.2^{\circ}$ after the osteotomy $(\mathrm{P}<0.001)$. The Mean \pm SD femoral neck-shaft angle was computed to be $121.9 \pm 22.8^{\circ}$ before and $144.5 \pm 17.7^{\circ}$ after the valgus osteotomy $(\mathrm{P}<0.001)$. Data were presented as Mean \pm SD or N (\%). DHS: Dynamic Hip Screw.

The radiologic sign of AVN was identified in 6 patients (Figure 1a). Surgical site infection did not occur in any of the explored patients. Six patients required revision sur-

Table 1. Characteristic of the patients with femoral neck non-union $(n=20)$

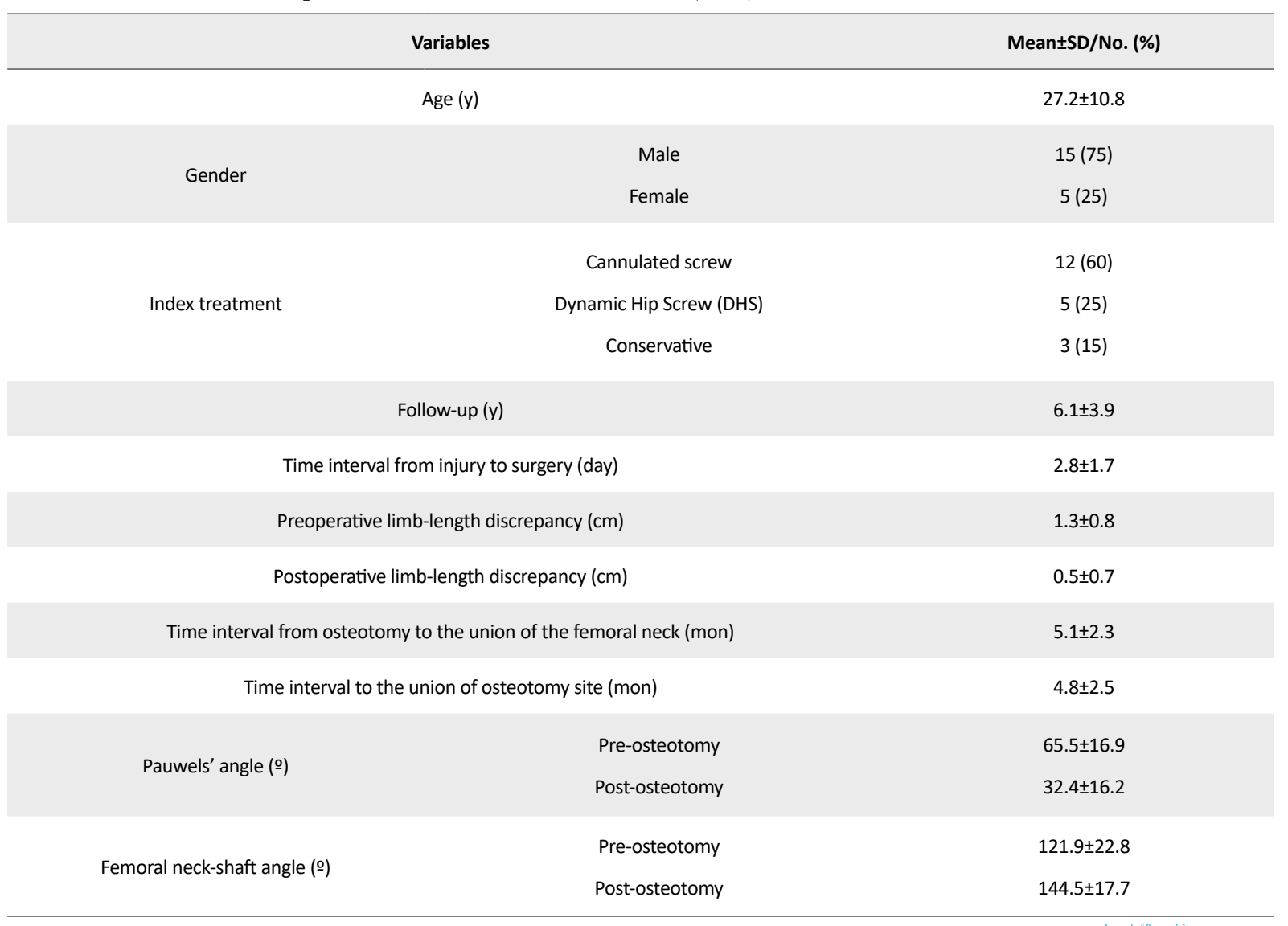



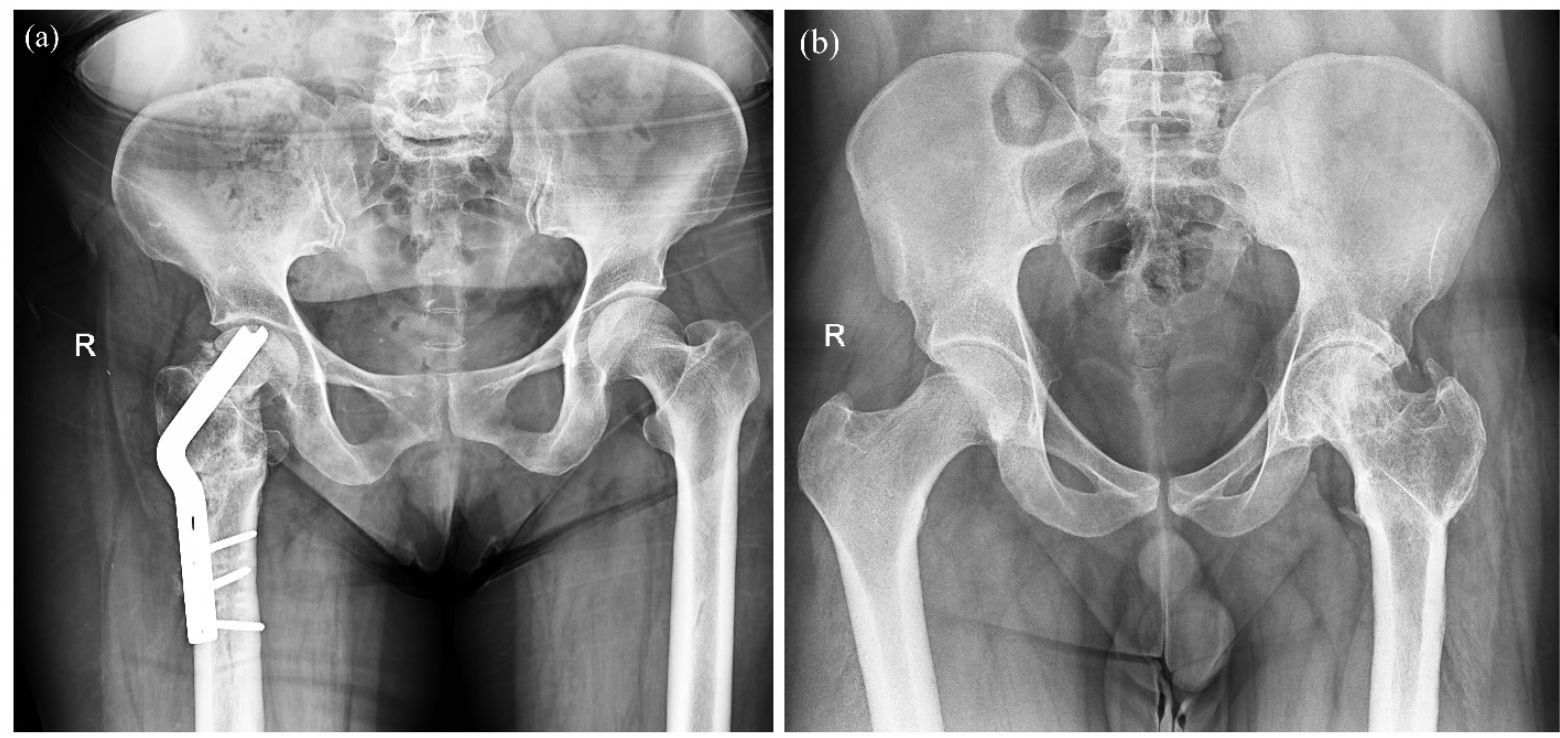

Orthopedic Science

Figure 1. Anteroposterior radiograph (a) and the radiologic sign of AVN (b)

Showing the irritation of the joint by the device two years after valgus osteotomy. The device was removed after one year due to the patient's complaint of feeling uncomfortable in the cold.

gery. The revision surgery was THA in 2 patients and device removal in 4 patients. The device removal was mainly because of joint irritation by the device (Figure 1b).

\section{Discussion}

The current study evaluated the safety and efficacy of valgus osteotomy in treating FNN. Based on our results, the union of the femoral neck was achieved in $18(90 \%)$ patients following the valgus osteotomy. Additionally, limb length discrepancy, Pauwels' angle, and neck-shaft angle were considerably improved in the study subjects. The radiologic sign of AVN was observed in $6(30 \%)$ cases. Moreover, $6(30 \%)$ patients required revision surgery, which included total hip replacement in 2 patients and device removal in 4.

The outcome of valgus osteotomy in managing FNN was reported in several studies. Schwartsmann et al. reported the outcome of valgus osteotomy in 42 patients with FNN and a mean age of 42.4 years. Valgus osteotomy led to the union of femoral neck in $38(90.4 \%)$ patients. The average neck-shaft angle increased from $132^{\circ}$ to $144^{\circ}$. Good to excellent functional outcomes were obtained in 34 (80.9\%) patients. Moreover, 6 (14.2\%) patients with unfavorable functional outcomes ended up with THA. Before the osteotomy, limb length discrepancy was present in 24 patients. Valgus osteotomy led to the equalization in 16 patients [7]. In the current study, the success rate of valgus osteotomy was measured as
90\%. The average neck-shaft angle improved from $121.9^{\circ}$ to $144.5^{\circ}$. Furthermore, $2(10 \%)$ required a THA.

Subash reported the outcomes of valgus osteotomy with DHS fixation in treating 15 patients with FNN and a mean age of 45.2 years. The mean follow-up period of the patients was 3 years. The union of all fractures was detected after 6 months. The mean Pauwels' angle and the neck-shaft angle were measured to be $34^{\circ}$ and $135.2^{\circ}$, respectively. Oxford score was improved from a mean preoperative value of 20.4 to a mean postoperative value of 37.9. The only observed complications were 2 cases of superficial skin infection [9]. The mean final Pauwels' angle and the neck-shaft angles were $32.4^{\circ}$ and $144.5^{\circ}$, respectively. That result was comparable with those of the study of Subash.

A large body of literate also reported the outcome of valgus osteotomy in treating FNN with a success rate of 74\%-100\% [4, 10-19]. Accordingly, the success rate of valgus osteotomy seems to be acceptable and consistent between different studies. The rate of conversion to THA is also comparable between studies; a conversion rate of $12 \%-18 \%$ was reported in this regard $[15,20]$. However, the rate of hip replacement is associated with the followup duration. This is because degenerative changes progress with time and total hip replacement could become necessary [7].

Altogether, valgus osteotomy could be regarded as a safe and efficient alternative for THA in treating FNN. 
THA provides earlier rapid pain relief and allows early mobilization; however, long-term results are not always as expected, particularly for young patients who outlive their disease. Therefore, valgus osteotomy, as a less radical approach, is worth considering.

\section{Conclusions}

Valgus osteotomy is a safe and efficient technique for managing FNN. It is associated with a high success rate and a small rate of complications. Moreover, the limb length discrepancy could be significantly resolved during the procedure. Therefore, valgus osteotomy could be regarded as an appropriate alternative for THA, particularly for young and active patients. The main limitation of the study was its retrospective design, i.e., potentially associated with the high risk of information bias. The retrospective design also prevented the evaluation of functional outcomes in this research.

\section{Ethical Considerations}

\section{Compliance with ethical guidelines}

The study was approved by the Review Board of Iran University of Medical Sciences.

\section{Funding}

This research did not receive any grant from funding agencies in the public, commercial, or non-profit sectors.

\section{Authors' contributions}

Conceptualization and Methodology: Kaveh Gharanizadeh; Writing - original draft: Hamed Tayyebi; Data collection: Abbas Esmaeli, Maziar Rajei, and Mohammadreza Heidarikhoo; Data analysis: Mehdi Siahpoosh.

\section{Conflict of interest}

The authors declared no conflicts of interest.

\section{References}

[1] Thorngren KG, Hommel A, Norrman PO, Thorngren J, Wingstrand $\mathrm{H}$. Epidemiology of femoral neck fractures. Injury. 2002; 33 (S3):C1-7. [DOI:10.1016/S0020-1383 (02)00324-8]

[2] Tabrizi A, Mehdizadeh M. Bilateral femoral neck stress fractures in a patient with severe coxa vara, morbid obesi- ty and osteomalacia: A case report. J Res Orthop Sci. 2019; 6(2):e84431. [DOI:10.5812/soj.84431]

[3] Pisoude K, Elahifar O, Sohrabi MB, Khajemozafari J. Comparison of proximal femur locking compression plate and intramedullary nailing in the treatment of sub-trochanteric fractures. J Res Orthop Sci. 2019; 6 (2):e91166. [DOI:10.5812/soj.91166]

[4] Mathews V, Cabanela ME. Femoral neck nonunion treatment. Clin Orthop Relat Res. 2004; (419):57-64. [PMID] [DOI:10.1097/00003086-200402000-00010]

[5] Raaymakers ELFB, Marti RK. Nonunion of the femoral neck: Possibilities and limitations of the various treatment modalities. Indian J Orthop. 2008; 42(1):13-21. [DOI:10.4103/00195413.38575]

[6] Pauwels F. Femoral neck fracture. A mechanical problem (chapter). In: Pauwels F. Biomechanics of the Locomotor Apparatus. Berlin: Springer Berlin Heidelberg; 2012. https:// www.google.com/books/edition/Biomechanics_of_the Locomotor_Apparatus/xL3uCAAAQBAJ?hl=en\&gbpv $=0$

[7] Schwartsmann CR, Spinelli LdF, Yépez AK, Boschin LC, Silva MF. Femoral neck nonunion treatment by valgus intertrochanteric osteotomy. Acta Ortop Bras. 2015; 23(6):319-22. ] [DOI:10.1590/1413-785220152306148750]

[8] Varghese VD, Livingston A, Boopalan PR, Jepegnanam TS. Valgus osteotomy for nonunion and neglected neck of femur fractures. World J Orthop. 2016; 7(5):301-7. [DOI:10.5312/ wjo.v7.i5.301]

[9] Subash Y. The role of valgus osteotomy with DHS fixation in the management of fracture nonunion of the neck of femur. Int J Res Orthop. 2018; 4(1):166-72. [DOI:10.18203/issn.24554510.IntJResOrthop20175666]

[10] Prakash J, Keshari V, Chopra RK. Experience of valgus osteotomy for neglected and failed osteosynthesis in fractures neck of femur. Int Orthop. 2020; 44:705-13. [DOI:10.1007/ s00264-019-04422-x]

[11] Wentzensen A, Weller S. [Pseudarthrosis as a complication of femoral neck fracture (German)]. Aktuelle Traumatol. 1983; 13(2):72-6. [PMID]

[12] Anglen JO. Intertrochanteric osteotomy for failed internal fixation of femoral neck fracture. Clin Orthop Relat Res. 1997; (341):175-82. [DOI:10.1097/00003086-199708000-00027]

[13] Zehi K, Bouguira A, Saadaoui F, Meherzi MH, Karray S, Zouari M, et al. [Valgus osteotomy in the treatment of pseudoarthrosis of the femoral neck: 41 cases (French)]. Rev Chir Orthop Reparatrice Appar Mot. 2001; 87(6):562-8. [PMID]

[14] Magu N, Singh R, Mittal R, Garg R, Wokhlu A, Sharma AK. Osteosynthesis and primary valgus intertrochanteric osteotomy in displaced intracapsular fracture neck of femur with osteoporosis in adults. Injury. 2005; 36(1):110-22. [DOI:10.1016/j.injury.2004.02.017]

[15] Wu C-C, Shih C-H, Chen W-J, Tai C-L. Treatment of femoral neck nonunions with a sliding compression screw: Comparison with and without subtrochanteric valgus osteotomy. J Trauma Acute Care Surg. 1999; 46(2):312-7. [DOI:10.1097/00005373-199902000-00019]

[16] Ballmer FT, Ballmer PM, Baumgaertel F, Ganz R, Mast JW. Pauwels osteotomy for nonunions of the femoral neck. 
Orthop Clin North Am. 1990; 21(4):759-67. [DOI:10.1016/ S0030-5898 (20)31516-9]

[17] Lies A, Scheuer I. [Pseudo-arthrosis of the neck of the femur in adults. Pathogenesis, therapy and results (German)]. Unfallheilkunde. 1983; 86(3):116-21. [PMID]

[18] Pidhorz L, Lahbabi S, Deburge A. [Pseudarthrosis of the femur neck. Treatment by intertrochanteric osteotomy (French)]. Rev Chir Orthop Reparatrice Appar Mot. 1974; 60(3):205-21. [PMID]

[19] Ghosh B, Bhattacharjya B, Banerjee K, Bera AK. Management of non-united neck femur fracture by valgus osteotomy: A viable alternative. J Indian Med Assoc. 2012; 110(11):819-20. [PMID]

[20] Min BW, Bae KC, Kang CH, Song KS, Kim SY, Won YY. Valgus intertrochanteric osteotomy for nonunion of femoral neck fracture. Injury. 2006; 37(8):786-90. [DOI:10.1016/j. injury.2006.02.045] [PMID] 